\title{
Facial Expression Recognition Using Fast Walidlet Hybrid Transform
}

\author{
W. A. Mahmoud ${ }^{1}$, J J. Stephan' ${ }^{2}$ A. A. Razzak ${ }^{3}$ \\ ${ }^{1}$ Professor of Digital Signal processing. University of Uruk. College of Engineering. Baghdad. Iraq \\ ${ }^{2}$ Iraqi Commission for Computers \& Informatics. Institute for Postgraduate Studies. Baghdad. Iraq. \\ ${ }^{3}$ Mustansiriya University. Faculty of Education. Department of Computer Science. Baghdad. Iraq.
}

profwaleed54@gmail.com

\begin{abstract}
Automatic analysis of facial expressions is rapidly becoming an area of intense interest in computer vision and artificial intelligence research communities. In this paper an approach is presented for facial expression recognition of the six basic prototype expressions (i.e., joy, surprise, anger, sadness, fear, and disgust) based on Facial Action Coding System (FACS). The approach is attempting to utilize a combination of different transforms (Walid let hybrid transform); they consist of Fast Fourier Transform; Radon transform and Multiwavelet transform for the feature extraction. Korhonen Self Organizing Feature Map (SOFM) then used for patterns clustering based on the features obtained from the hybrid transform above. The result shows that the method has very good accuracy in facial expression recognition. However, the proposed method has many promising features that make it interesting. The approach provides a new method of feature extraction in which overcome the problem of the illumination, faces that varies from one individual to another quite considerably due to different age, ethnicity, gender and cosmetic also it does not require a precise normalization and lighting equalization. An average clustering accuracy of $94.8 \%$ is achieved for six basic expressions, where different databases had been used for the test of the method.
\end{abstract}

Crossref $10.36371 /$ port.2020.3.4

Keywords: Facial expression recognition; relative geometric position; Dependency; hybrid feature.

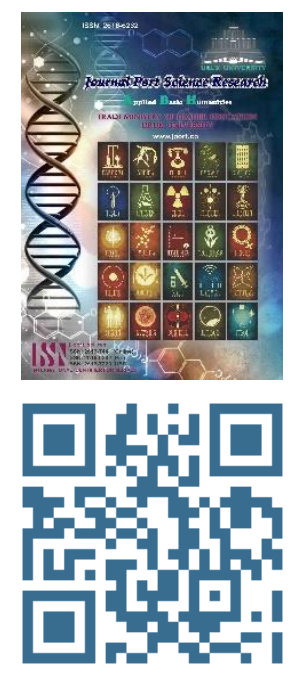

\section{INTRODUCTION}

Human beings are capable of communicating with each other in many different ways. The most common methods exploited for this include the use of words, gestures and facial expressions, either individually or in some combination. Using facial expressions has to be one of the most complicated forms, if not the most complicated form. Much can be communicated by even a single facial expression, and hence these expressions have become a very important aspect of our communication. Consequently, it is only natural that the demand for a computer system that can recognize human facial expressions has arisen.

It is argued that to truly achieve effective humancomputer intelligent interaction (HCII), there is a need for the computer to be able to interact naturally with the user, similar to the way human-human interaction takes place. Humans interact with each other mainly through speech, but also through body gestures, to emphasize a certain part of the speech and display of emotions. One of the important ways humans display emotions is through facial expressions.

Automatic facial expression analysis is a complex task as the topology of faces varies from one individual to another quite considerably due to different age, ethnicity, gender, facial hair, cosmetic and occluding objects such as glasses and hair. Further, faces appear disparate because of pose and lighting changes. Variation such as these have to be addressed at different stages of an automatic facial expression analysis system, such as normalization task including (pose and illumination), and face segmentation task including (background and facial feature separation).

Automatic analysis of facial expressions is rapidly becoming an area of intense interest in computer vision and artificial intelligence research communities. Automated systems that sense, process, and interpret human facial expressions have important commercial potential; they seem to have a natural place in commercial products such as computer systems for video conferencing, video telephony, video surveillance, video indexing [1-4], robotics as well as virtual reality, image understanding, psychological studies, facial nerve grading in medicine [5-6], face image compression and synthetic face animation [7].

Furthermore, monitoring and interpreting facial expressions are important to lawyers, the police, and security agents, who are often interested in issues concerning deception and attitude. Facial expression 
intensities may measure by determining either geometric deformations of facial features or the density of wrinkles appearing in certain face regions. For example, the degree of smiling is communicated by the magnitude of cheek and lip corner rising as well as wrinkle displays. Since there are inter-personal variations with regard to the amplitudes of facial actions, it is difficult to determine absolute facial expression intensities, without referring to the neutral face of a given subject. Note that measuring the intensity of spontaneous facial expressions is more difficult than measuring posed facial expression, which are usually displayed with an exaggerated intensity and can thus be identified more reliably [8].

There is one main methodological approach of how to measure the facial expressions, this is the FACS (Facial Action Coding System), which was developed by (Ekman and Friesen [9]) and has been considered as a foundation for describing facial expressions.

Neural networks were used for facial expression classification [13-19] and were either applied directly on face images [20,21] or combined with facial features extraction and representation methods such as PCA (Principal Component Analysis), ICA (Independent Component Analysis). The former are unsupervised statistical analysis methods that allow for a considerable dimensionality reduction, which both simplifies and enhances subsequent classification. These methods have been employed both in a holistic manner [14, 24, 25] as well as locally using mosaic-like patches extracted from small facial regions $[13,10,26,32]$.

It is well known that wavelet-based image representation has many advantages and there is strong evidence that the human visual system processes images in a multi scale way according to psycho visual research. Thus, spatial frequency preprocessing of faces is justified by what is known about early visual processing. By spatial frequency analysis, an image is represented as a weighted combination of basic functions, in which high frequencies carry finely, detailed information and low frequencies carry coarse, shape-based information. Recently, there have been renewed interests in applying wavelet and Gabor wavelet techniques to solve many real-world problems and in image processing and computer vision in particular. Examples include image database retrieval [26] and face recognition [11], [21], [33] facial expression recognition [22], [25], [26], [27].

An appropriate wavelet transform can result in robust representations with regard to lighting changes and be capable of capturing substantial facial features while keeping computational complexity low.

In this paper an approach is presented for facial expression recognition of the six basic prototype expressions (i.e., joy, surprise, anger, sadness, fear, and disgust) based on Facial Action Coding System (FACS). The approach is implementing the Walidlet Hybrid Transform [28-31] for the feature extraction and then using Kohonen Self Organizing Feature Map (SOFM) [11], [12] for pattern clustering to cluster the discriminated information vectors extracted from its matrix coefficients.

\section{THE PROPOSED APPROACH}

Recently Walidlet Hybrid Transform is used in image enhancement, so a new interest is rising on this topic. The main reason is that a complete framework has been recently building (Raghad A. [28]) particular for what concerns the construction of hybrid transforms for efficient feature representation of image signals. The main characteristic of walidlet is the possibility to provide a multiresolution analysis of the image in the form of coefficient matrices. Strong arguments for the use of multiresolution decomposition can be found in psychovisual research, which offer evidence that the human visual system process the images in multiscale way.

The Walidlet transform starts by apply the two dimensions Fast Fourier Transform (2-D FFT) on the two dimensions signal (image). The Next step is to map a line sampling scheme into a point sampling scheme using the Radon transform. Hence, it is required to take the onedimension inverse Fast Fourier Transform (1-D IFFT) for each column of the produced two dimensions signal. Finally, the Multiwavelet transform performed on each row of the resultant two dimensions signal. The structure of the Walidlet transform is given in (Figure 1).

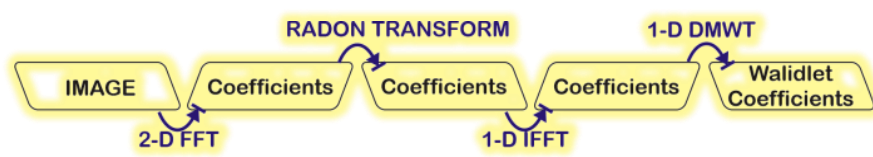

Figure (1) Flow diagram of Walidlet Transform

\section{ALGORITHM FOR COMPUTATION OF WALIDLET TRANSFORM}

The following are the Walidlet Transform's computation steps:

Step (1): Resizing: To implement the Radon Transform and the best sequence of directions in the following steps, a two-dimensional signal must be first converted into a prime square form. zero padding operations should be performed on the image (adding rows or columns of zero values), if the image is not a square matrix. 
Step (2): Computation of two-Dimensional Fourier Transform; the two-dimensional Fast Fourier transform should be applied in this step, to the resized twodimensional signal, and store the resultant coefficients in fx matrix, this process is necessary to obtain the DC Component.

Step (3): DC value replacement; the first element of the produced transformed matrix is representing the DC value of the two-dimensional signal. This value will be replaced with a zero value.

Step (4): Computation of the Radon Transform; to compute the Radon transform, the next steps should be performed:

A) Computation of the Best Sequence of Directions.

B) Rearranging Fourier Slices.

Step (5): Compute the One Dimension Inverse Fast Fourier Transform (1-D IFFT), here the one-dimension inverse Fast Fourier transform should be applied to each column of the produced matrix from step (5).

Step (6): Resizing of the 1-D IFFT Resultant Matrix, it is necessary here to resize the vectors into a power of two elements in order to enable the computation of last step.

Step (7): Compute the One Dimension Multiwavelet Transform (1-D DMWT), The final step of the Walidlet transform is to apply the one-dimension Multiwavelet transform for each row of the produced matrix from step (6). Figure (2), exhibit the flowchart of the proposed transform (Walidlet Transform).

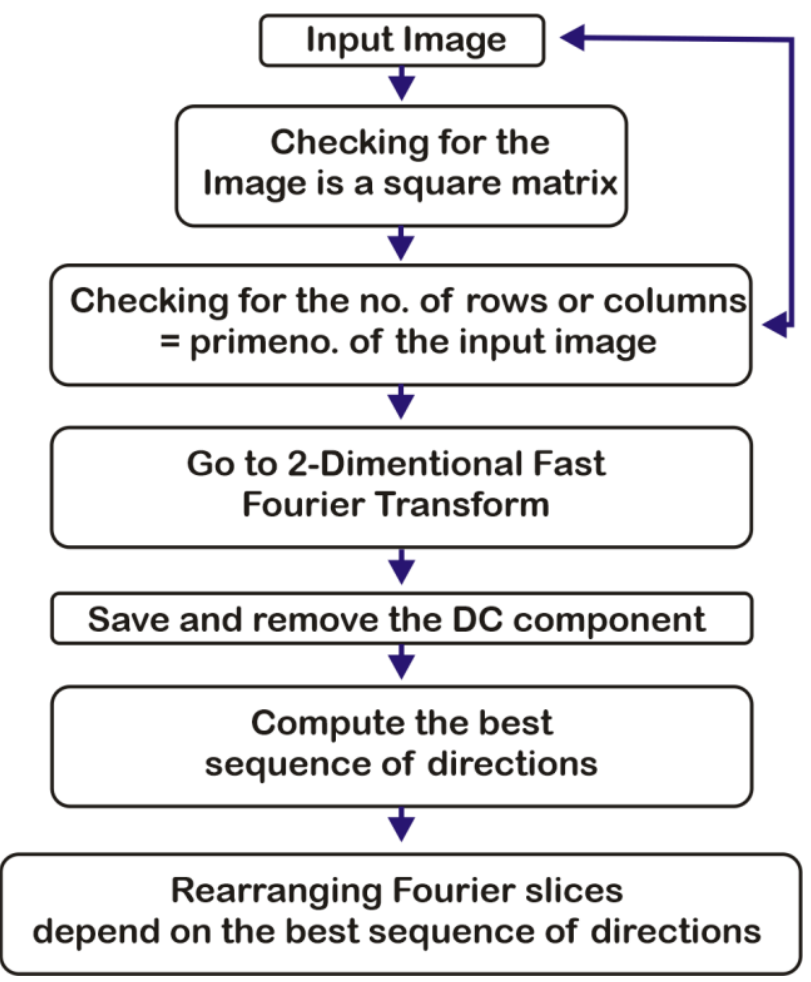

Figure 2 Flowchart of proposed transform (Walidlet Transform)

\section{FEATURE VECTORS EXTRACTION}

Before proceeding with feature extraction, an approximate normalization step is performed on the images selected from the "Cohn-Kanade [AU-Coded] Facial Expression Database" [34] database, regarding the translation, rotation and scaling and no lighting equalization is performed. After this step of preprocessing, the Walidlet hybrid transform is performed on the whole face image but the walidlet coefficients will be considered only in the column wise aspect. The first four columns of the coefficient's matrix is considered (Low resolution information). There is no need to consider more columns because, the size of the coefficients is becoming too large and no more voluble information is obtained. Therefore, the face image is described by 256 walidlet coefficients vector which represent reasonable amount of information. It is important to take decisions only on the most essential, so-called discriminatory information, which is conveyed by the extracted features. Each of the 256 coefficient vectors contains information about the face expression.

\section{FEATURE VECTORS CLUSTERING}

When solving a pattern recognition problem, the ultimate objective is to design a recognition system which will classify unknown patterns with the lowest possible probability of misrecognition. It is well known that, as the complexity of a classifier grows rapidly with the number of dimensions of the pattern space. Thus, the problem is how to face the dimensionality reduction? An efficient way of reducing dimensionality and clustering expression information is to compute the topological relationships between these feature vectors. Thus, Self-Organizing Map neural network is used, the dimensionality is reduced to two dimensions instead of 256. Also, the discriminated information regarding the face expressions is presented on the Self-Organizing feature Map in terms of the topological relationships.

\section{DISCRIMINATE FEATURE'S VECTORS OF WALIDLET TRANSFORM}

Several experiments had been performed to decide which columns among the first four coefficients' columns of walidlet hybrid transform may hold the most discriminated information. Self-Organizing Map neural network is used to show the topological relationships between these feature vectors regarding all the facial expression images' data collected from "Cohn-Kanade [AU-Coded] Facial Expression Database" [26], as shown in (Figures 3-8). Only two columns are found to be the most significant feature vectors, these

columns are (column 1 and 3), the other two (columns 2 and 4) are found less significant. 
The experiments are performed as follow:

1) A collection of (149) FACS based expression images are chosen from "Cohn-Kanade" database for the six basic facial expressions, with a varying number of expression images for each expression type as shown in (Table 1). The images are chosen with an acceptable combination of AUs with respect to each prototype expression or its major variants combinations.

Table 1 Number of images chosen for each expression.

\begin{tabular}{|c|c|c|c|c|c|c|}
\hline Anger & Disgust & Fear & Happy & Sad & Surprise & Total \\
\hline 18 & 29 & 15 & 34 & 27 & 26 & 149 \\
\hline
\end{tabular}

2) Pre-processing operations (approximate normalization) performed on this collection regarding the shift, scale and rotation.

3) The Walidlet Hybrid Transform is applied on these collected images individually to obtain the coefficient matrix for each single image. An ensemble of walidlet coefficients are shown in (Figure11).

4) Only the first four column of the coefficient's matrix are extracted for each image of the total 149 collected images and then save these column's data in different files as shown in (Table 2).

Table 2 Number of files for each column/expression for the 149 images.

\begin{tabular}{|l|c|c|c|c|c|c|}
\hline & Anger & Disgust & Fear & Happy & Sad & Surprise \\
\hline Column1 & 18 & 29 & 15 & 34 & 27 & 26 \\
\hline Column2 & 18 & 29 & 15 & 34 & 27 & 26 \\
\hline Column3 & 18 & 29 & 15 & 34 & 27 & 26 \\
\hline Column4 & 18 & 29 & 15 & 34 & 27 & 26 \\
\hline
\end{tabular}

5) For each column, all the coefficients' files for all expressions are added to construct a total file for each column.

6) In order to cluster the coefficient vectors for all expressions and for each column, the Self-Organizing Map neural network is used in which considered an unsupervised classification method, where no predefined classes and training examples are involved. The SOFM is applied on each column's total file constructed in step 5 . The clustering of the images' coefficients such that a similar coefficient vectors are grouped together and dissimilar coefficient vectors are grouped into other clusters. The SOFM applied on each column (i.e., four independent SOFM runs, one for each column). The summary of SOFM parameters used are shown in (Table 3 ) and the topological relationships between the facial expression coefficient vectors for column $1 \& 3$ are shown in (Figures 9\& 10).

Table 3 Parameters used in SOFM for the Classification task.

\begin{tabular}{|c|c|c|c|}
\hline Pattern Vector Size & No. Of Patterns & Map size & Accepted Error \\
\hline 256 & 149 & $35 \times 35$ & 1. E-7 \\
\hline
\end{tabular}

7) In order to determine the ability of the Walidlet Hybrid Transform in capturing the facial expression image's discriminatory information, the code-book vectors (every node in the map may be thought of as code-book vector) are compared against each input vector (149 image vectors). Since the labels of the input vectors are arranged in ranges according to the six expressions as shown in (Table 4), then every node on the map is labeled by assigning it the label of the input vector most similar to it (difference value between the two vectors is less than 1.E-7). This allows the topological structure of the map to be examined as shown in Figures 9\&10). Also, different colors are assigned for each expression node label range as shown in (Table 4).

Table 4 Ranges of image labels and colors assigned for each expression on the SOFM maps.

\begin{tabular}{|l|l|l|l|l|l|l|}
\hline Expression & Anger & Disgust & Fear & Happy & Sad & Surprise \\
\hline Ranges & $0-17$ & $18-46$ & $47-61$ & $62-95$ & $96-122$ & $123-148$ \\
\hline Colors & Blue & Orange & Yellow & Green & Purple & Red \\
\hline
\end{tabular}

8) By the visual inspection to the maps presented in (Figures $9 \& 10$ ), it can be observed that, the most significant vectors are the first and the third ones, since SOFM is able to groups similar expression nodes in clusters (several clusters for each class) for each expression. For columns two and four, SOFM is not able to cluster similar expression nodes in clusters for each expression. The nodes are dispersing across the map without any explicit fashion that governing their distribution. For this reason, columns (1\&3) can be considered the most significant vectors and they capture the discriminated information regarding the six facial expressions.

9) Now to decide which column of the two most significant coefficient vectors (column $1 \& 3$ ) is more significant, a comparison is made based on the number of clusters for each class (one class for each expression), the clustering accuracy described by equation (7) below and the expressions overlapping as described in (Sec. 7) below .

The clustering accuracy is calculated according to the following rule:

(NumberOfExpressionNodes) - (NumberOfNonClusterNodes) (NumberOfExpressionNodes)

The detail clustering information are presented in (Table 5\&6) for columns $1 \& 3$ correspondingly. The results of (Tables 5, 6) shows that, column one is more significant in capturing the discriminated information than column three, since the average clustering accuracy of column one $(94.8 \%)$ is pretty higher than the average accuracy of column three (84.8\%), also the expressions overlapping are insignificant in column's one in contrast with column three as shown in (Tables 8 \& 9).

It can be observed from the results presented in the first column's map and as shown in (Figure 9) that, Walidlet Transform possess the following properties: 
1) Robust against the illumination problem in the images, the illumination is filtered out and illuminated images used in the experiment are well clustered within their similar expression groups. In (Table 7) examples are given to illustrate this property.

2) Partially side view face is well clustered within its expression group, as shown in (Figure 6, Image node \#71). The method still capable of capturing the expression features without losing important information with this side view pose.

3) Facial hair especially in the forehead area of the face did not mix up with the expression features of the face as shown in (Table 7), so the feature vector is still posses the expression information with different fashions of forehead hair .

4) Gender, race and ethnicity factors are filtered out by the transform in the low-resolution information and they did not affect the expression feature vectors, so that the expression nodes are clustered mainly according to the facial expressions.

5) The hard expressions to perform by the subjects (Fear, Anger and Sad) as they described by some researchers, and difficult to recognize by other approaches are well separated and clustered especially for the anger and sad expressions.

6) Full head view with hair style images as shown in (Table 7) are still well clustered within their similar expression, so that it may be possible to process the facial expression without any cropping is necessary. This point may need further investigation in an independent research .

7) The subject's identities are filtered out by the transform, so that different expressions images for the same person are well clustered within their similar expression groups (Figures 4 - 8). This property considered most important in facial expression recognition, since the identity features could overcome the expression features for the same subject. This may lead to cluster all the images of the same person in one group regardless of what his expression is.

Table 5 Classes and their Clustering information for Column one.

\begin{tabular}{|c|c|c|c|}
\hline & $\begin{array}{c}\text { No. of } \\
\text { Clusters } \\
\text { for each } \\
\text { Class }\end{array}$ & $\begin{array}{c}\text { No. of non-clustered } \\
\text { expression nodes }\end{array}$ & $\begin{array}{c}\text { Clustering } \\
\text { Accuracy }\end{array}$ \\
\hline Anger & 2 & 1 & $95 \%$ \\
\hline Disgust & 3 & 2 & $97 \%$ \\
\hline Fear & 4 & none & $100 \%$ \\
\hline Happy & 5 & 6 & $84 \%$ \\
\hline Sad & 4 & None & $100 \%$ \\
\hline Surprise & 3 & 2 & $93 \%$ \\
\hline Total & 21 & 10 & $94.8 \%$ \\
\hline
\end{tabular}

Table 6 Classes and their Clustering information for Column one.

\begin{tabular}{|c|c|c|c|}
\hline & $\begin{array}{c}\text { No. of Clusters } \\
\text { for each Class }\end{array}$ & $\begin{array}{c}\text { No. of non-clustered } \\
\text { expression nodes }\end{array}$ & $\begin{array}{c}\text { Clustering } \\
\text { Accuracy }\end{array}$ \\
\hline Anger & 2 & 3 & $84 \%$ \\
\hline Disgust & 3 & 9 & $69 \%$ \\
\hline Fear & 2 & 4 & $74 \%$ \\
\hline Happy & 5 & 9 & $74 \%$ \\
\hline Sad & 3 & 2 & $93 \%$ \\
\hline Surprise & 4 & 2 & $93 \%$ \\
\hline Total & 19 & 29 & $81.2 \%$ \\
\hline
\end{tabular}

Table 7 Labels of SOFM's expression images and their associated obstacles.

\begin{tabular}{|c|c|c|}
\hline $\begin{array}{c}\text { Illuminated } \\
\text { faces }\end{array}$ & $\begin{array}{c}\text { Full head view } \\
\text { with hair style }\end{array}$ & Forehead hair \\
\hline$\# 0, \# 2, \# 12, \# 21$, & & $\# 1, \# 10, \# 13$, \\
$\# 26, \# 40, \# 48$, & & $\# 26, \# 40, \# 84$, \\
$\# 50, \# 54, \# 55, \# 66$, & $\# 82, \# 84, \# 96$, & $\# 98, \# 111, \# 122$, \\
$\# 67, \# 69, \# 70, \# 75$, & $\# 100, \# 114$, & $\# 126, \# 138, \# 142$, \\
$\# 76, \# 79, \# 83, \# 88$, & $\# 134, \# 140, \# 141$ & $\# 145$ \\
$\# 92, \# 94, \# 98, \# 111$, & & \\
$\# 121, \# 125, \# 137$, & & \\
$\# 128, \# 139, \# 140$ & & \\
\hline
\end{tabular}

\begin{tabular}{|c|c|c|c|c|c|}
\hline S34-03 & $\begin{array}{c}\mathrm{S} 42- \\
04\end{array}$ & $\begin{array}{c}\text { S89- } \\
03\end{array}$ & $\begin{array}{c}\text { S100- } \\
05\end{array}$ & $\Longrightarrow$ & $\begin{array}{c}\text { S134- } \\
03\end{array}$ \\
\hline \#0 & $\# 1$ & $\# 2$ & $\# 3$ & \#4 & $\# 5$ \\
\hline S10-04 & $\begin{array}{c}\text { S11- } \\
04\end{array}$ & $\begin{array}{c}\text { S14- } \\
03\end{array}$ & $\begin{array}{c}\text { S22- } \\
05\end{array}$ & S37-03 & $\begin{array}{c}\text { S50- } \\
04\end{array}$ \\
\hline \#6 & \#7 & \#8 & $\# 9$ & $\# 10$ & \#11 \\
\hline & $\begin{array}{c}\text { S53- } \\
03\end{array}$ & $\begin{array}{c}\text { S92- } \\
03\end{array}$ & $\begin{array}{c}\text { S109- } \\
03\end{array}$ & $=$ & $\begin{array}{c}\text { S117- } \\
06\end{array}$ \\
\hline \#12 & $\# 13$ & $\# 14$ & $\# 15$ & $\# 16$ & \#17 \\
\hline
\end{tabular}

Figure 3 Anger facial images collected from "Cohn-Kanade [26]" Database with their labels on the maps.

Note: the images displayed are the ones with consent for publication; other images are not allowed to be displayed according to the agreement with the owner of the database, (Cohn-Kanade [26]). Therefore, only their actual labels in the database are shown.

\begin{tabular}{|c|c|c|c|c|c|}
\hline $\begin{array}{c}\text { S46- } \\
04\end{array}$ & S87-04 & S77-07 & S74-04 & $\begin{array}{c}\text { S11- } \\
05\end{array}$ & S14-04 \\
\hline$\# 18$ & $\# 19$ & $\# 20$ & $\# 21$ & $\# 22$ & $\# 23$ \\
\hline $\begin{array}{c}\text { S26- } \\
04\end{array}$ & S32-05 & S42-05 & S56-02 & $\begin{array}{c}\text { S96- } \\
03\end{array}$ & S97-04 \\
\hline
\end{tabular}




\begin{tabular}{|c|c|c|c|c|c|}
\hline$\# 24$ & $\# 25$ & $\# 26$ & $\# 27$ & $\# 28$ & $\# 29$ \\
\hline $\begin{array}{c}\text { S98- } \\
03\end{array}$ & S109-04 & $\Rightarrow$ & S19-04 & $\begin{array}{l}\text { S75- } \\
04\end{array}$ & $\Leftrightarrow$ \\
\hline$\# 30$ & \#31 & \#32 & \#33 & $\# 34$ & \#35 \\
\hline $\begin{array}{c}\text { S22- } \\
06\end{array}$ & S45-05 & S69-03 & S73-06 & $\begin{array}{c}\text { S76- } \\
05\end{array}$ & S88-04 \\
\hline$\# 36$ & $\# 37$ & $\# 38$ & $\# 39$ & $\# 40$ & $\# 41$ \\
\hline $\begin{array}{l}\text { S105 } \\
-08\end{array}$ & & S107-05 & & $\begin{array}{c}\text { S116 } \\
-06\end{array}$ & \\
\hline$\# 42$ & \#43 & $\# 44$ & \#45 & $\# 46$ & \\
\hline
\end{tabular}

Figure 4 Disgust facial images collected from "CohnKanade [26]" Database.

\begin{tabular}{|c|c|c|c|c|c|}
\hline $\begin{array}{l}\text { S11- } \\
03\end{array}$ & S62-01 & $\begin{array}{l}\text { S65- } \\
02\end{array}$ & (0) & $\begin{array}{l}\text { S88- } \\
02\end{array}$ & S10-01 \\
\hline$\# 47$ & $\# 48$ & $\# 49$ & $\# 50$ & $\# 51$ & $\# 52$ \\
\hline $\begin{array}{c}\text { S50- } \\
01\end{array}$ & S59-02 & $\begin{array}{l}\text { S91- } \\
01\end{array}$ & S107-04 & $\begin{array}{l}\mathrm{S} 124 \\
-03\end{array}$ & \\
\hline$\# 53$ & $\# 54$ & $\# 55$ & $\# 56$ & $\# 57$ & \#58 \\
\hline $\begin{array}{c}\text { S119 } \\
-04\end{array}$ & $\Rightarrow$ & $\begin{array}{l}\mathrm{S} 138 \\
-01\end{array}$ & & & \\
\hline$\# 59$ & \#60 & \#61 & & & \\
\hline
\end{tabular}

Figure 5 Fear facial images collected from "Cohn-Kanade [26]" Database.

\begin{tabular}{|c|c|c|c|c|c|}
\hline S81-02 & $\begin{array}{c}\text { S10- } \\
06\end{array}$ & $\begin{array}{c}\text { S14- } \\
05\end{array}$ & S32-06 & S35-06 & $\begin{array}{c}\text { S42- } \\
06\end{array}$ \\
\hline \#62 & \#63 & \#64 & $\# 65$ & \#66 & \#67 \\
\hline S50-06 & $\begin{array}{c}\text { S53- } \\
04\end{array}$ & $\begin{array}{c}\text { S60- } \\
02\end{array}$ & S63-02 & S68-02 & $\begin{array}{c}\text { S71- } \\
05\end{array}$ \\
\hline \#68 & \#69 & $\# 70$ & $\# 71$ & $\# 72$ & $\# 73$ \\
\hline S75-06 & $\begin{array}{c}\text { S86- } \\
02 \\
\end{array}$ & $\begin{array}{c}\text { S91- } \\
03 \\
\end{array}$ & S98-04 & $\begin{array}{c}\text { S116- } \\
07 \\
\end{array}$ & $\begin{array}{c}\text { S89- } \\
02 \\
\end{array}$ \\
\hline$\# 74$ & $\# 75$ & $\# 76$ & $\# 77$ & $\# 78$ & \#79 \\
\hline S68-01 & $\begin{array}{c}\text { S11- } \\
06\end{array}$ & $\begin{array}{c}\text { S26- } \\
06\end{array}$ & S34-05 & S37-06 & $\begin{array}{c}\text { S46- } \\
05\end{array}$ \\
\hline$\# 80$ & \#81 & $\# 82$ & $\# 83$ & $\# 84$ & $\# 85$ \\
\hline - 19 & $\begin{array}{c}\text { S61- } \\
05\end{array}$ & $\begin{array}{c}\text { S64- } \\
03\end{array}$ & S69-04 & S72-06 & $\begin{array}{c}\text { S76- } \\
06\end{array}$ \\
\hline \#86 & \#87 & $\# 88$ & $\# 89$ & $\# 90$ & \#91 \\
\hline
\end{tabular}

\begin{tabular}{|c|c|c|c|c|c|}
\hline S83-03 & $\begin{array}{c}\text { S87- } \\
05\end{array}$ & $\begin{array}{c}\text { S96- } \\
04\end{array}$ & $\begin{array}{c}\text { S109- } \\
06\end{array}$ & & \\
\hline$\# 92$ & $\# 93$ & $\# 94$ & $\# 95$ & & \\
\hline
\end{tabular}

Figure 6 Happy facial images collected from "CohnKanade [26]" Database

\begin{tabular}{|c|c|c|c|c|c|}
\hline S105-08 & S14-01 & S37-02 & $\mathrm{S} 50-03$ & & S71-02 \\
\hline$\# 96$ & \#97 & \#98 & \#99 & \#100 & $\# 101$ \\
\hline S82-02 & S113-03 & S116-05 & S130-04 & $\ldots$ & S122-04 \\
\hline$\# 102$ & $\# 103$ & $\# 104$ & $\# 105$ & \#106 & $\# 107$ \\
\hline S11-02 & S26-02 & \begin{tabular}{|l} 
S32-02 \\
\end{tabular} & S42-02 & S45-02 & S46-01 \\
\hline$\# 108$ & $\# 109$ & $\# 110$ & $\# 111$ & $\# 112$ & \#113 \\
\hline 5 & S68-01 & S72-02 & S81-02 & S87-02 & S115-06 \\
\hline \#114 & $\# 115$ & $\# 116$ & $\# 117$ & $\# 118$ & \#119 \\
\hline S117-02 & S131-02 & \begin{tabular}{|l|} 
S136-03 \\
\end{tabular} & \begin{tabular}{|l|} 
S117-02 \\
\end{tabular} & & \\
\hline$\# 120$ & $\# 121$ & $\# 122$ & $\# 120$ & & \\
\hline
\end{tabular}

Figure 7 Sad facial images collected from "Cohn-Kanade [26]" Database.

\begin{tabular}{|c|c|c|c|c|c|}
\hline $\begin{array}{c}\text { S14- } \\
01\end{array}$ & S32-01 & $\begin{array}{c}\text { S35- } \\
01\end{array}$ & $\begin{array}{c}\text { S42- } \\
01\end{array}$ & $\mid \begin{array}{c}\text { (ब) (3) } \\
\cdots \\
\pi\end{array}$ & $\begin{array}{c}\text { S58- } \\
01\end{array}$ \\
\hline$\# 123$ & $\# 124$ & $\# 125$ & $\# 126$ & \#127 & $\# 128$ \\
\hline $\begin{array}{c}\text { S63- } \\
01\end{array}$ & S78-01 & $\begin{array}{c}\text { S81- } \\
01\end{array}$ & $\begin{array}{c}\text { S100- } \\
02\end{array}$ & S122-01 & $\begin{array}{c}\text { S10- } \\
02\end{array}$ \\
\hline$\# 129$ & $\# 130$ & $\# 131$ & $\# 132$ & $\# 133$ & $\# 134$ \\
\hline $\begin{array}{c}\text { S11- } \\
01\end{array}$ & S26-01 & $\begin{array}{c}\text { S34- } \\
01\end{array}$ & $\begin{array}{c}\text { S37- } \\
01\end{array}$ & S44-01 & $\begin{array}{c}\text { S46- } \\
01\end{array}$ \\
\hline$\# 135$ & $\# 136$ & $\# 137$ & $\# 138$ & $\# 139$ & $\# 140$ \\
\hline $\begin{array}{c}\text { S50- } \\
01\end{array}$ & S53-01 & $\begin{array}{c}\text { S61- } \\
01\end{array}$ & $\begin{array}{c}\text { S65- } \\
01\end{array}$ & S74-02 & $\begin{array}{c}\text { S75- } \\
02\end{array}$ \\
\hline$\# 141$ & $\# 142$ & $\# 143$ & $\# 144$ & $\# 145$ & $\# 146$ \\
\hline $\begin{array}{c}\text { S80- } \\
01\end{array}$ & & & & & \\
\hline$\# 147$ & \#148 & & & & \\
\hline
\end{tabular}

Figure 8 Surprise facial images collected from "CohnKanade [26]" Database. 


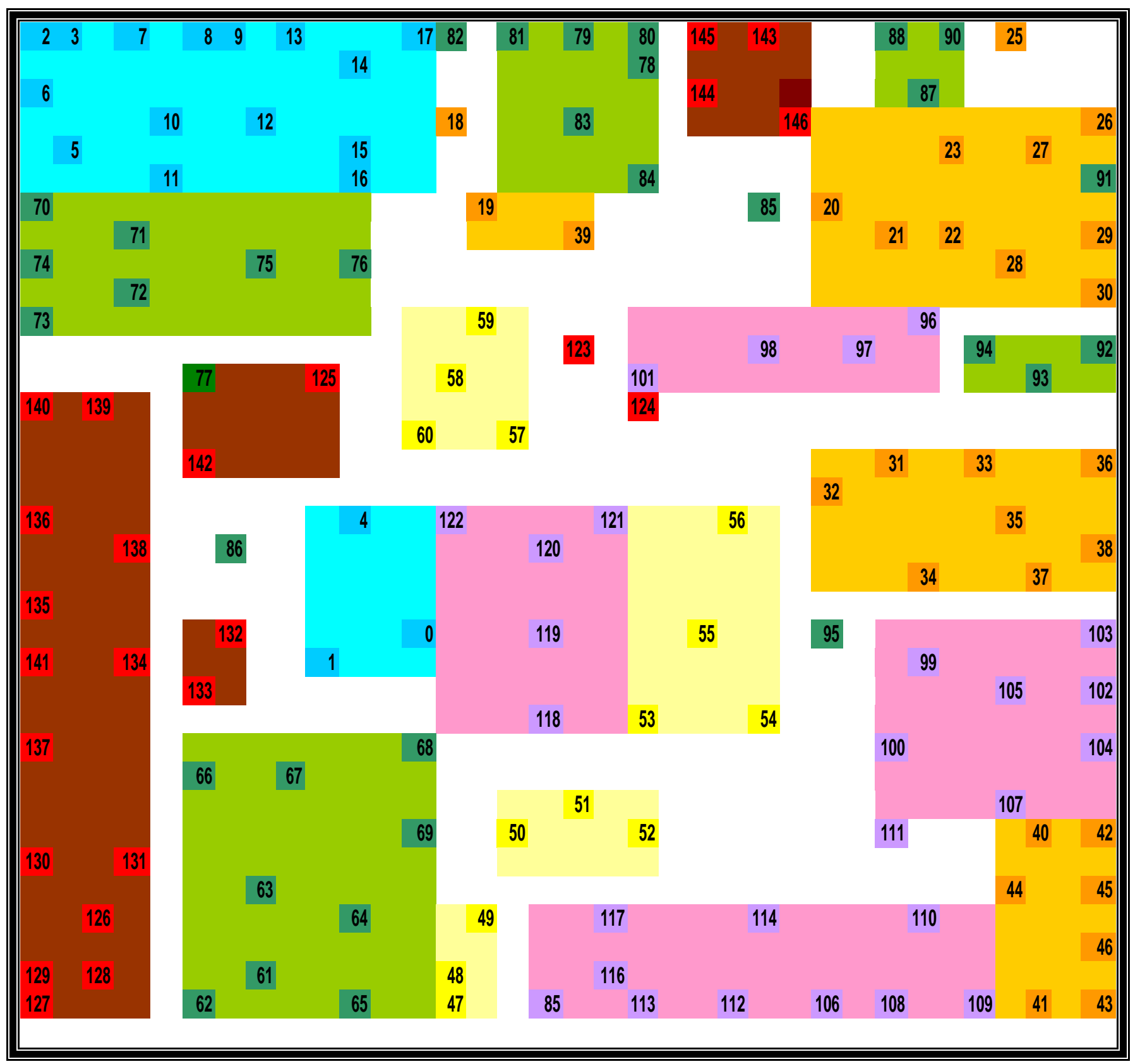

Figure 9 Expression nodes clusters on SOFM's map for 149 expression images collected from "Cohn-Kanade" database using Walidlet Transform coefficients of column one.

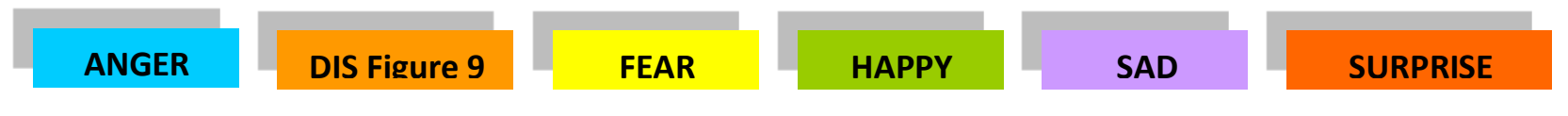




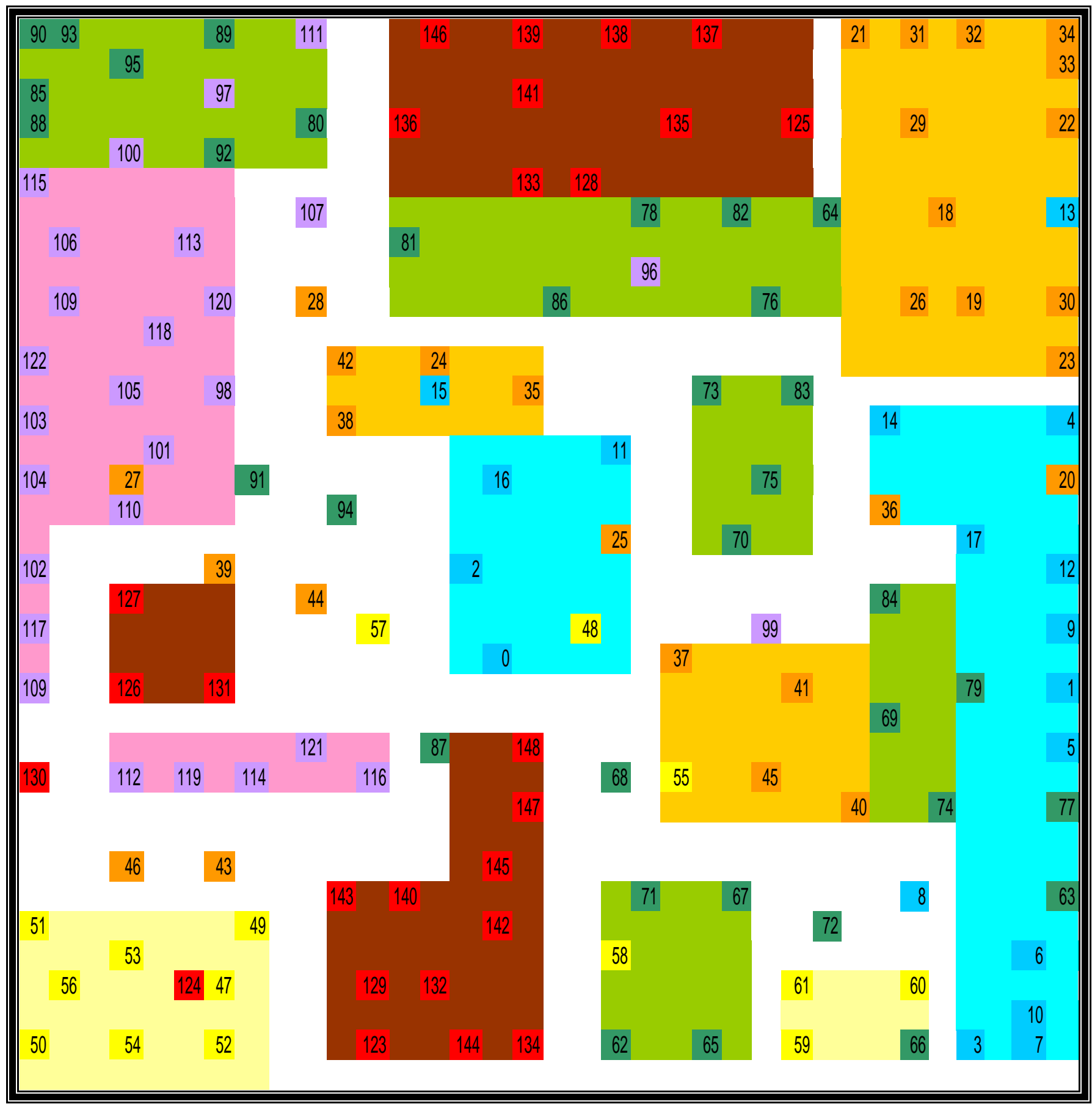

Figure 10 Expression nodes clusters on SOFM's map for 149 expression images collected from "Cohn-Kanade" database using Walidlet Transform coefficients of column three.

\begin{tabular}{|c|c|c|c|c|c|}
\hline ANGER & DISGUST & FEAR & HAPPY & SAD & SURPRISE \\
\hline
\end{tabular}




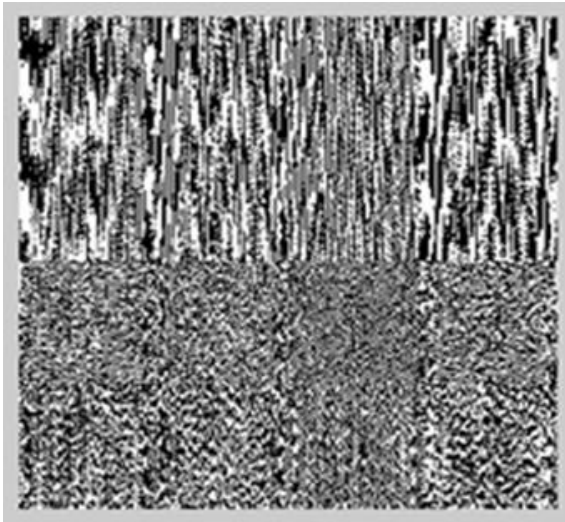

Figure 11 Ensemble of Walidlet Hybrid Transform

\section{CROSS VALIDATION OF ALL EXPRESSIONS TESTS}

A cross validation made between all the expression to explore the overlapping recognition between any expressions in favors of the others for the first and third column, (Tables 8 \& 9) shows these overlapping between all these expressions.

From the analysis of the results of the tables $(8 \& 9)$, it can be observed that, column one's coefficients are more reliable in separating the types of expressions than third column's coefficients. In column one, only the happy expression is overlapped with surprise and disgust expressions in a minimum rate for both expressions (i.e., 0.029 ) which considered insignificant rates. While in column three all expressions are overlapped in favors of the others also with minimum overlapping rates.

Table 8 Overlapping recognitions results between expressions for the first column.

\begin{tabular}{|l|l|l|l|l|l|l|}
\hline & HAPPY & SURPRISE & DISGUST & FEAR & SAD & ANGER \\
\hline HAPPY & 1.0 & 0.029 & 0.029 & 0.0 & 0.0 & 0.0 \\
\hline SURPRISE & 0.0 & 1.0 & 0.00 & 0.00 & 0.0 & 0.0 \\
\hline DISGUST & 0.0 & 0.0 & 1.0 & 0.0 & 0.0 & 0.0 \\
\hline FEAR & 0.0 & 0.0 & 0.0 & 1.0 & 0.0 & 0.0 \\
\hline SAD & 0.0 & 0.0 & 0.0 & 0.0 & 1.0 & 0.0 \\
\hline ANGER & 0.0 & 0.0 & 0.0 & 0.0 & 0.0 & 1.0 \\
\hline
\end{tabular}

Table. 9 overlapping recognitions results between expressions for the third column.

\begin{tabular}{|l|l|l|l|l|l|l|}
\hline & $\begin{array}{l}\text { HAPP } \\
\mathrm{Y}\end{array}$ & $\begin{array}{l}\text { SURPRIS } \\
\mathrm{E}\end{array}$ & $\begin{array}{l}\text { DISGUS } \\
\mathrm{T}\end{array}$ & $\begin{array}{l}\text { FEA } \\
\mathrm{R}\end{array}$ & $\begin{array}{l}\mathrm{SAD} \\
\mathrm{R}\end{array}$ & $\begin{array}{l}\text { ANGE } \\
\mathrm{R}\end{array}$ \\
\hline HAPPY & 1.0 & 0.0 & 0.0 & 0.029 & 0.0 & 0.088 \\
\hline $\begin{array}{l}\text { SURPRIS } \\
\text { E }\end{array}$ & 0.0 & 1.0 & 0.0 & 0.038 & 0.0 & 0.0 \\
\hline DISGUST & 0.0 & 0.0 & 1.0 & 0.0 & $\begin{array}{l}0.03 \\
4\end{array}$ & 0.0 \\
\hline FEAR & 0.0 & 0.0 & 0.067 & 1.0 & 0.0 & 0.0 \\
\hline SAD & 0.148 & 0.0 & 0.0 & 0.0 & 1.0 & 0.0 \\
\hline ANGER & 0.0 & 0.0 & 0.11 & 0.0 & 0.0 & 1.0 \\
\hline
\end{tabular}

2. CONCLUSIONS

This paper addressed the problem of automatic facial expression recognition based on FACS AUs. An approach utilizes walidlet transform on static images of facial expressions is used in order to capture the discriminated information of the six basic prototypes facial expressions basic expression. The SOFM neural network is used to cluster the feature vectors extracted from Walidlet coefficients, using a feature map of (35X35) expression nodes. Only low-resolution information are extracted from its coefficient matrix, these information may lies in one or more of its first four coefficient vectors. Research shown that, the first and the third columns are most significant in their discriminated power and in particular, the first column .

The test showed an average clustering accuracy of $95 \%$ was achieved for the first column, and $81.2 \%$ for the third column. It can be concluded from these results that, walidlet coefficients of low resolution can be used as a discriminated features for the task of facial expression recognition with a very high percent of accuracy .

The interesting feature of this transform is its ability in capturing the facial expression with an approximate normalization in which considered an excellent achievement since the process of precise normalization is quit expensive task regarding the time and efforts. Even with an automatic normalization a human subject must interfere at one or more stages of the process. This transform also possesses other strong characteristic in capturing facial expression features with multi-state face or nearly front-view faces. Also, the transform shows a strong capability in filtering out the illumination that occurs in images. Based on the work in this

\section{REFERENCES}

[1] Liu, C., Hirota, K., Ma, J., Jia, Z., \& Dai, Y. (2021). Facial Expression Recognition Using Hybrid Features of Pixel and Geometry. IEEE Access, 9, 18876-18889. https://doi.org/10.1109/ACCESS.2021.3054332

[2] Dulguerov, P., Marchal, F., Wang, D., \& Gysin, C. (1999). Review of objective topographic facial nerve evaluation methods. American Journal of Otology, 20(5), 672-678.

[3] Shanthi, P., \& Nickolas, S. (2021). An efficient automatic facial expression recognition using local neighborhood feature fusion. Multimedia Tools and Applications, 80(7), 10187-10212. https://doi.org/10.1007/s11042-020-10105-2

[4] Chen, J., Chen, Z., Chi, Z., \& Fu, H. (2018). Facial Expression Recognition in Video with Multiple Feature Fusion. IEEE Transactions on Affective Computing, 9(1), 38-50. https://doi.org/10.1109/TAFFC.2016.2593719

[5] Li, Y., Zeng, J., Shan, S., \& Chen, X. (2019). Occlusion Aware Facial Expression Recognition Using CNN With Attention Mechanism. IEEE Transactions on Image Processing, 28(5), 2439-2450. https://doi.org/10.1109/TIP.2018.2886767

[6] Choi, K., Chen, J., Park, M. W., Yang, H., Choi, W., Ikonin, S., ... Park, J. (2020). Video Codec Using Flexible Block Partitioning and Advanced Prediction, Transform and Loop Filtering Technologies. IEEE Transactions on Circuits and Systems for Video Technology, 30(5), 1326-1345. https://doi.org/10.1109/TCSVT.2020.2971268 
[7] Fasel, B., \& Luettin, J. (2003). Automatic facial expression analysis: A survey. Pattern Recognition. Elsevier Ltd. https://doi.org/10.1016/S0031-3203(02)00052-3

[8] Ekman, P., \& Friesen, W. V. (1971). Constants across cultures in the face and emotion. Journal of Personality and Social Psychology, 17(2), 124-129. https://doi.org/10.1037/h0030377

[9] Darwin, C. (1873). The Expression of the Emotions in Man and Animals. The Journal of the Anthropological Institute of Great Britain and Ireland, 2, 444. https://doi.org/10.2307/2841467

[10] Ekman, P. (2012). Emotional and conversational nonverbal signals. In Gesture, Speech, and Sign (pp. 45-55). Oxford University Press. https://doi.org/10.1093/acprof:oso/9780198524519.003.0003

[11] Kanade, T., Cohn, J. F., \& Tian, Y. (2000). Comprehensive database for facial expression analysis. In Proceedings - 4th IEEE International Conference on Automatic Face and Gesture Recognition, FG 2000 (pp. 46-53). IEEE Computer Society. https://doi.org/10.1109/AFGR.2000.840611

[12] Ekman, P., \& Friesen, W. V. (1978). Manual for the facial action coding system. Consulting Psychologist, 104, 0.

[13] Oatley, K., \& Johnson-Laird, P. N. (2014, March). Cognitive approaches to emotions. Trends in Cognitive Sciences. https://doi.org/10.1016/j.tics.2013.12.004

[14] Fellenz, W. A., Taylor, J. G., Tsapatsoulis, N., \& Kollias, S. (1999). Comparing template-based, feature-based and supervised classification of facial expressions from static images. Computational Intelligence and Applications, 354-359.

[15] Padgett, C., \& Cottrell, G. (1997). Representing face images for emotion classification. In Advances in Neural Information Processing Systems (pp. 894-900). Neural information processing systems foundation.

[16] Bartlett, M. S. (2001). Face Image Analysis by Unsupervised Learning. Face Image Analysis by Unsupervised Learning. Springer US. https://doi.org/10.1007/978-1-4615-1637-8

[17] Pantic, M., \& Rothkrantz, L. J. M. (2000). Expert system for automatic analysis of facial expressions. Image and Vision Computing, 18(11), 881-905. https://doi.org/10.1016/S0262-8856(00)00034-2

[18] Kulkarni, S. S., Reddy, N. P., \& Hariharan, S. I. (2009). Facial expression (mood) recognition from facial images using committee neural networks. BioMedical Engineering Online, 8. https://doi.org/10.1186/1475-925X-8-16

[19] Lisetti, C. L., \& Rumelhart, D. E. (1998). Facial Expression Recognition using a Neural Network. In Proceedings of the 11th International Flairs Conference (FLAIRS-98). Menlo Park, CA: AAAI Press.

[20] Kobayashi, H., \& Hara, F. (1997). Facial interaction between animated 3D face robot and human beings. In Proceedings of the IEEE International Conference on Systems, Man and Cybernetics (Vol. 4, pp. 3732-3737). IEEE. https://doi.org/10.1109/icsmc.1997.633250

[21] Yoneyama, M., Iwano, Y., Ohtake, A., \& Shirai, K. (1997). Facial expressions recognition using discrete Hopfield neural networks. In IEEE International Conference on Image Processing (Vol. 1, pp. 117-120). IEEE Comp Soc. https://doi.org/10.1109/icip.1997.647398

[22] Pumlumchiak, T., \& Vittayakorn, S. (2017). Facial expression recognition using local Gabor filters and PCA plus LDA. In 2017 9th International Conference on Information Technology and Electrical Engineering, ICITEE 2017 (Vol. 2018-January, pp. 1-6). Institute of Electrical and Electronics Engineers Inc. https://doi.org/10.1109/ICITEED.2017.8250446

[23] Hasan. H. M., AL.Jouhar, W, A., Alwan, M. A., (2012). 3-D Face Recognition Using Improved 3D Mixed Transform. International Journal of Biometrics and Bioinformatics (IJBB), Volume (6): Issue $\quad$ (1): 2012. https://citeseerx.ist.psu.edu/viewdoc/download?doi=10.1.1.740.1016\&rep=rep1\&type=pdf

[24] Belhumeur, P. N., Hespanha, J. P., \& Kriegman, D. J. (1997). Eigenfaces vs. fisherfaces: Recognition using class specific linear projection. IEEE Transactions on Pattern Analysis and Machine Intelligence, 19(7), 711-720. https://doi.org/10.1109/34.598228

[25] Bartlett, M. S., Littlewort, G., Braathen, B., Sejnowski, T. J., \& Movellan, J. R. (2003). A prototype for automatic recognition of spontaneous facial actions. In Advances in Neural Information Processing Systems. Neural information processing systems foundation.

[26] Bartlett, M. S., Braathen, B., Littlewort-ford, G., Hershey, J., Fasel, I., Marks, T., ... Movellan, J. R. (2001). Automatic Analysis of Spontaneous Facial Behavior: A Final Project Report. Cognitive Science, 1-39. 
[27] Lyons, M. J., Budynek, J., Plantey, A., \& Akamatsu, S. (2000). Classifying facial attributes using a 2-D Gabor wavelet representation and discriminant analysis. In Proceedings - 4th IEEE International Conference on Automatic Face and Gesture Recognition, FG 2000 (pp. 202-207). IEEE Computer Society. https://doi.org/10.1109/AFGR.2000.840635

[28] Aladdin Jassim, R. Amin Mahmoud, W. (2005). A Proposed Walidlet Transform With Its Application In Image Enhancement. Master's thesis, University of Technology - Institute of Informatics for Graduate Studies. Baghdad. https://iqdr.iq/search?view=98adaf824adb11ebb86a77c772d4f5b2

[29] Al-Jouhar, W. (2011). A Smart Single Matrix Realization of Fast Walidlet Transform. International Journal of Research and Reviews in $\begin{array}{llllll}\text { Computer } & \text { Science, } & \text { Vol. } & 1, & \text { issue } & 2,\end{array}$ https://www.researchgate.net/publication/323906455_A_Smart_Single_Matrix_Realization_of_Fast_Walidlet_Transform

[30] Al-Jouhar, W. A., M. Al-Talib, T. M. J. A., \& Salman, H. A. R. (2010). Fingerprint image recognition using Walidlet transform. Australian Journal of Basic and Applied Sciences, 4(8), 3970-3976.

[31] Al-Helali, A. H. M., Ali, H. A., Al-Dulaimi, B., Alzubaydi, D., \& Mahmmoud, W. A. (2009). Slantlet transform for multispectral image fusion. Journal of Computer Science, 5(4), 263-269. https://doi.org/10.3844/jcs.2009.263.269

[32] Lien, J. J., Cohn, J. F., Kanade, T., \& Li, C. C. (1998). Automated facial expression recognition based on FACS action units. In Proceedings - 3rd IEEE International Conference on Automatic Face and Gesture Recognition, FG 1998 (pp. 390-395). IEEE Computer Society. https://doi.org/10.1109/AFGR.1998.670980

[33] Al-Jouhar, W. A., Kadhim, D,J,. (2013). A Proposal Algorithm to Solve Delay Constraint Least Cost Optimization Problem. Journal of Engineering. Volume 19, Issue 1, Pages 155-160. https://www.iasj.net/iasj/article/63899

[34] Kanade, T., Cohn, J. F., \& Tian, Y. (2000). Comprehensive database for facial expression analysis. In Proceedings - 4th IEEE International Conference on Automatic Face and Gesture Recognition, FG 2000 (pp. 46-53). IEEE Computer Society. https://doi.org/10.1109/AFGR.2000.840611 